\title{
Estudio preliminar de las propiedades psicométricas de la escala de Funcionamiento Psicológico Positivo para una muestra de adolescentes uruguayos
}

\section{Preliminary study of the psychometric properties of the Positive Psychological Functioning scale for a sample of Uruguayan adolescents}

\section{Estudo preliminar das propriedades psicométricas da escala Positive Psychological Functioning para uma amostra de adolescentes uruguaios}

\author{
Mayda Portela, ORCID 0000-0001-7947-0563 \\ Universidad Católica del Uruguay
}

\begin{abstract}
Resumen: El objetivo del presente trabajo fue evaluar las propiedades psicométricas de la escala de Funcionamiento Psicológico Positivo (FPP) en adolescentes uruguayos. La escala está compuesta por 33 ítems que miden 11 recursos psicológicos. El FPP se constituye en un constructo de segundo orden del bienestar eudaimónico. Se realizó un estudio de tipo instrumental con una muestra por conveniencia conformada por 183 adolescentes (de 12 a 17 años) de población general. Los resultados de los análisis factoriales realizados resultan en una estructura de siete dimensiones: autoestima, autonomía, propósito vital, optimismo/disfrute, curiosidad, humor y dominio del entorno (RMSEA = .09, CFI= .99, TLI= .97), y adecuados valores de fiabilidad. La solución factorial organizada en siete factores explica el 62,15\% de la varianza. Estos resultados concuerdan con el único estudio publicado en población adolescente, que a diferencia del original y los posteriores replicados, utilizaron muestras de universitarios.
\end{abstract}

Palabras clave: funcionamiento psicológico positive; análisis factorial; propiedades psicométricas

Abstract: The objective of this paper was to evaluate the psychometric properties of the Positive Psychological Functioning scale (PPF) in Uruguayan adolescents. The scale is composed of 33 items that measure 11 psychological resources. The PPF constitutes a second-order construct of eudaimonic well-being. An instrumental study was carried out using a convenience sample made up of 183 adolescents (ages 12 through 17) from the general population. The results of the factor analysis carried out had a seven-dimensional structure: self-esteem, autonomy, purpose in life, optimism/enjoyment, curiosity, sense of humor, and environmental mastery (RMSEA $=.09, \mathrm{CFI}$ $=.99, \mathrm{TLI}=.97)$, and adequate reliability values. The factorial solution organized into seven factors explains $62.15 \%$ of the variance. These results coincide with the only published study for an adolescent population, which differs from the original and subsequent studies given that these analyzed samples from university students.

Keywords: positive psychological functioning; factor analysis; psychometric properties 
Resumo: O objetivo deste trabalho foi avaliar as propriedades psicométricas da escala de Funcionamento Psicológico Positivo (FPP) em adolescentes uruguaios. A escala é composta por 33 itens que medem 11 recursos psicológicos. O FPP constitui um construto de segunda ordem do bem-estar eudaimônico. Realizou-se estudo instrumental com amostra de conveniência composta por 183 adolescentes (12 a 17 anos) da população geral. Os resultados da análise fatorial realizada resultam em uma estrutura de sete dimensões: autoestima, autonomia, resiliência, otimismo, curiosidade, prazer e domínio do ambiente (RMSEA $=0,09, \mathrm{CFI}=0,99, \mathrm{TLI}=0,97)$ e valores de confiabilidade adequados. A solução fatorial organizada em sete fatores explica $62,15 \%$ da variância. Esses resultados são consistentes com o único estudo publicado em uma população de adolescentes que, ao contrário do original e das réplicas subsequentes, utilizou amostras de estudantes universitários.

Palavras-chave: funcionamento psicológico positivo; análise fatorial; propriedades psicométricas

Recibido: $1 / 04 / 2020$

Aceptado: 8/02/2021

Cómo citar:

Portela, M. (2021). Estudio preliminar de las propiedades psicométricas de la escala de Funcionamiento Psicológico Positivo para una muestra de adolescentes uruguayos. Ciencias Psicológicas, 15(1), e-2396. doi: https://doi.org/10.22235/cp.v15i1.2396

Correspondencia: Mayda Portela. Universidad Católica del Uruguay.E-mail: mportela@ucu.edu.uy

La adolescencia es aquella etapa del ciclo vital caracterizada por procesos de maduración biológica y desarrollo psicológico, cognitivo y social que permiten el paso desde la niñez hacia la adultez (Gaete, 2015). Una de las principales características de esta etapa es la búsqueda de la identidad, la motivación por la pertenencia a un grupo de pares y cierto movimiento de independencia de su grupo familiar. En este proceso normal del desarrollo se producen varios cambios en diferentes áreas de la vida, resultando importante medir los aspectos saludables con los que los adolescentes cuentan.

Los recursos psicológicos son factores preponderantes en tanto aspectos que indican las aptitudes para el desempeño, así como el ajuste a las diferentes situaciones de vida, ya que con ellas las personas se regulan a sí mismas y a sus relaciones con el entorno (Alvaro et al., 2010).

En este sentido, los procesos de despliegue psicológico se activan, dependiendo de las capacidades de cada uno para hacer frente a situaciones estresantes tal como lo desarrolla la teoría de Conservación de los Recursos de Hobfoll (1989). Esta teoría establece que los recursos psicológicos se instrumentan tanto frente a la percepción de amenaza, como a la percepción de pérdida de recursos, entendiendo la pérdida real como fuente de estrés. Las personas están sometidas a permanente demanda del entorno y necesidad de regulación, adaptación, y ajuste al mismo. Los recursos se instrumentan tanto para hacer frente a situaciones de estrés como para la persecución de metas deseadas; constituyen aspectos positivos de la personalidad. De acuerdo con la teoría de Hobfoll (2002), se establece que la activación de los recursos permite una mejor adaptación al entorno y que, por otra parte, estos recursos no son independientes, sino que provoca una activación en cadena; es decir, la estimulación de un recurso desencadena la activación de otros. Varios estudios se apoyan en esta teoría (González, Andrade \& Jiménez, 1997; Rueda \& Pérez, 2005; Vázquez, Rivera \& Quintanilla, 2011).

Siguiendo esta línea, el modelo de Funcionamiento Psicológico Positivo (FPP) ofrece una perspectiva y marco teórico-conceptual relevante para entender las diferencias individuales en lo 
relativo a recursos psicológicos, tanto en evaluación diagnóstica como en trabajo terapéutico, siendo que los recursos psicológicos son pasibles de ser evaluados y entrenados.

Desde el punto de vista conceptual, los antecedentes del FPP se vinculan a la perspectiva del bienestar eudaimónico, por un lado y a la teoría de Conservación de los Recursos por el otro. El bienestar se conceptualiza a partir de dos aspectos: subjetivo o hedónico, relacionado con la felicidad y el psicológico o eudaimónico, cuyo desarrollo teórico se centra en el potencial de las personas y su ajuste al medio (Muratori, Zubieta, Ubillos, González, \& Bobowik, 2015). En este sentido, Robert Cumminis, autor de referencia en la temática, desarrolla la teoría homeostática del bienestar, recogiendo y vinculando el concepto de homeostasis para representar el manejo del estado del bienestar. Conceptualiza que hay una disposición general de los sujetos a mantener niveles de bienestar subjetivo dentro de determinado rango (Tomyn, Weinberg, \& Cummins, 2015). Esta conceptualización plantea que cuando falla la homeostasis, ocasionada por la presencia de un desafío importante negativo, las personas experimentan un afecto negativo que si perdura en el tiempo se convierte en una condición crónica. No obstante, si las exigencias exteriores son intensas, puede afectar la homeostasis y el hecho de que se nivele o se cronifique dependerá de los recursos personales con los que se cuente para adaptarse a las circunstancias (Tomyn et al., 2015).

Por otra parte, el modelo del FPP fue desarrollado a partir de la teoría de Conservación de los Recursos cuyo aporte reside en definir a los recursos psicológicos como características de personalidad que son valiosas en sí mismas ya que se vinculan con la obtención de resultados positivos. Favorecen la consecución de logros y metas en cuanto permiten a los individuos adaptarse al entorno, y finalmente son estables y pueden ser aprendidas (Hobfoll, 1989). De acuerdo con el autor, los recursos suelen estar relacionados, formando conglomerados de modo que la obtención de un recurso favorece la activación de otro, y el mismo proceso se da ante una pérdida de recurso (a este concepto lo llamó caravanas de recursos).

Carol Ryff, por su parte, desarrolló un modelo multidimensional sobre el bienestar psicológico, el cual está formado por los recursos que siguen: autoaceptación, relaciones positivas con otros, autonomía, dominio del entorno, propósito en la vida y crecimiento personal (Ryff, 1989). Este modelo es consonante con el anterior en cuanto también propone que los recursos se relacionan entre sí. La presencia o desarrollo de los recursos psicológicos así entendidos aportan al óptimo funcionamiento de la persona, permiten hacer frente a las situaciones internas y externas, incrementan la fortaleza individual, familiar y social y, en definitiva, permiten un estado de bienestar desde la perspectiva eudaimónica (Padrós \& Rivera, 2014).

El constructo Funcionamiento Psicológico Positivo surge a partir de una reciente investigación de la Universidad Complutense de Madrid compuesta por dos estudios. El primero de ellos tuvo por objetivo describir cómo están organizados los recursos psicológicos (autonomía, resiliencia, autoestima, propósito en la vida, goce, optimismo, curiosidad, creatividad, humor, dominio ambiental y vitalidad). El segundo estudio se propuso replicar los resultados del estudio 1 y poner a prueba la escala. Los resultados indicaron que existe una interconexión entre los recursos psicológicos, que forman un constructo de segundo orden que dieron en llamar FPP (Merino \& Privado, 2015).

El FPP es definido como "una molécula compuesta de átomos interconectados (recursos psicológicos) cuya contribución varía en función de su magnitud (carga del factor)" (Merino \& Privado, 2015, p. 52). Por tanto, el concepto conecta el funcionamiento psicológico, los recursos, las fortalezas y debilidades, así como la tendencia de la magnitud de factores. Así, el FPP se constituye en un constructo de segundo orden del bienestar eudaimónico, dando la idea de una tendencia disposicional de funcionamiento psicológico positivo, de acuerdo con el peso de los factores de recursos psicológicos. La definición del FPP, por tanto, sería la disposición de funcionamiento positivo de la persona compuesta por la movilidad de los factores positivos que la integran, cuya particularidad es que está siendo activada por la fuerza o debilidad de los recursos que la componen. 
La escala que evalúa el FPP está compuesta por 33 ítems que se responden en una escala tipo Likert del 1 a 5, donde 1 es completamente en desacuerdo y 5 es completamente de acuerdo. Las dimensiones de la escala están compuestas de la siguiente manera: autoestima (ítems 1, 18, 20); resiliencia (ítems 2, 14, 25); optimismo (ítems 3, 15, 21); creatividad (ítems 4, 9, 12); autonomía $(5,8,10)$; domino del entorno $(6,22,31)$; vitalidad $(7,19,32)$; propósito vital $(11,24$, 26); humor $(13,23,29)$; disfrute $(16,28,33)$; curiosidad $(17,27,30$; el ítem 22 está invertido).

Los datos originales que reportan los autores son que la carga del factor de segundo orden sobre los factores de primer orden es $>.74$ y cargas de factores de primer orden en los elementos $>$.60. Las cargas de los factores resultaron ser estadísticamente significativas $(\mathrm{p}<.001)$. El valor RMSEA fue de .06, indicando buen ajuste a la estructura factorial propuesta. El índice de ajuste incremental muestra moderado ajuste, NFI valor fue de .702 y CFI fue de .878. El índice de ajuste de parsimonia tiene valores superiores a .50, PNFI .644, por lo que también se muestra buen ajuste de los datos al modelo (Merino \& Privado, 2015).

Posteriormente se realizó una investigación en la ciudad de México para validar la escala del FPP. Los datos indican que la fiabilidad de la prueba es adecuada con un $\alpha$ de Cronbach de .70. La subescala que presenta menor fiabilidad es la de resiliencia (.56). La puntuación total de la escala (que incluye esta subescala) presentó una fiabilidad adecuada (.91). El NFI obtenido fue de .88 y el CFI de .92, por lo que el modelo presentaría un ajuste moderado (Merino, Privado, \& Gracia, 2015).

Por otra parte, se ha publicado un estudio de adaptación y validación de la escala en población universitaria portuguesa. La misma estuvo compuesta por 1131 estudiantes universitarios con una media de edad de 19.6. La misma informó de buenas propiedades psicométricas del FPP, donde la mayoría de las subescalas presentan un $\alpha$ de Cronbach $>.60$ y el total de la escala un $\alpha$ de .90 , concluyendo que la escala presenta niveles confiables para su uso en la población de estudio (Oliveira, Merino, Privado, \& Almeida, 2017).

Recientemente se ha publicado un estudio de validación de la escala de FPP en México, con una muestra de 313 adolescentes cuya media de edad fue de 12.7 años. Esta investigación mostró una estructura factorial de 4 factores, que en su conjunto explican el 56,83\% de la varianza total. El factor 1 está conformado por 10 ítems que corresponden a las dimensiones autoestima y resiliencia. El factor 2 pertenece a la dimensión disfrute, compuesto por 8 ítems. En el caso del factor 3, se integraron 6 ítems, que pertenecen a la dimensión optimismo; y el factor 4, con 5 ítems, es la dimensión autonomía-vitalidad. Se conservaron 29 de los 33 ítems originales, eliminando los ítems 8, 9, 10 y 22 (González, Torres, González, \& Ruiz, 2018).

Si observamos los estudios de validación publicados, tanto el original como los realizados en las ciudades de México y Portugal, que sostienen la distribución de la escala en 11 factores, estudiaron población universitaria; mientras que el último, que arroja una estructura factorial diferente (de 4 factores), se realizó con una muestra adolescente, similar a la del presente estudio instrumental.

\section{Método}

\section{Participantes}

La muestra se conformó por un total de 183 adolescentes uruguayos, de 12 a 17 años, pertenecientes a la población general, con una media de edad de 15 años. El 49,2 \% de los participantes eran de la capital, mientas que el 50,8 \% provenían del interior del país. El 54,6\% eran mujeres y el 45,4 \% del género masculino. El reclutamiento de los participantes se llevó a cabo a través de un muestreo no probabilístico, a partir del método de bola de nieve.

\section{Instrumentos}

La escala de Funcionamiento Psicológico Positivo (Merino \& Privado, 2015) está compuesta por 33 ítems que responden a una escala tipo Likert del 1 a 5, donde 1 es completamente 
en desacuerdo y 5 es completamente de acuerdo. La versión original se encuentra en español y mide 11 dimensiones: autoestima, resiliencia, optimismo, creatividad, autonomía, domino del entorno, vitalidad, propósito vital, humor, disfrute y curiosidad.

Luego del contacto y autorización de la autora original de la escala (Merino \& Privado, 2015) para su utilización en el presente estudio, se llevó a cabo una adaptación linguística de la escala, considerando la posibilidad de que los regionalismos locales sugirieran la conveniencia de modificar algún ítem o palabra. Se administró la escala a 15 adolescentes uruguayos, estudiantes de una institución de educación secundaria de la capital cuya lengua madre era el castellano y residían en Uruguay. Se evaluó la equivalencia conceptual y semántica.

Se evaluó que el lenguaje fuera claro, que la redacción fuera adecuada y que los ítems del cuestionario fueran comprensibles de acuerdo a tres categorías: 1. si se entiende el concepto general de lo que expresa el ítem; 2. que analicen palabra por palabra de la redacción de cada ítem observando si se comprende; 3. que aporten cualquier modificación de palabras que sean comprendidas de mejor manera por sus pares (adolescentes).

Los participantes sugirieron:

- en el ítem 6, cambiar la palabra compagino por combino;

- en el ítem 9 modificar la palabra dispares por diferente o distintas; se elige tomar la palabra diferente por el hecho de que es sugerida por más participantes;

- en el ítem 11, modificar la palabra entregado por enfocado;

- en el ítem 22 entienden que es más adecuado el uso de la palabra estudios por trabajo y "novio/a por pareja;

- en el ítem 31, modificar la palabra agobios por cansancio.

Cuatro meses más tarde se realiza la reaplicación de los inventarios con la incorporación de las modificaciones propuestas y solicitándose la misma consigna: a cada ítem de cada uno de los inventarios le corresponden tres columnas (concepto del ítem-palabra por palabraobservaciones). Esta aplicación se realiza con un total de 12 participantes, de la misma institución educativa, no presentando ninguna corrección ni modificación tanto de la comprensión general del ítem como de palabra por palabra, se resuelve incorporar estas modificaciones.

\section{Procedimiento y cuestiones éticas}

Se solicitó el acceso al contacto de adolescentes a través de instituciones de educación secundaria privada de la capital y el interior. La participación de los mismos fue autorizada por los familiares responsables por medio de las vías de comunicación interna de cada institución. En los liceos de Montevideo, la toma de la muestra fue presencial, mientras que en los departamentos del interior del país se realizó por medio digital.

La participación fue voluntaria y los adolescentes no obtuvieron ninguna compensación por dicha participación. Se procedió a digitalizar la escala para ser respondida vía web. Tanto en la versión digital como presencial, se dejó clara la consigna respecto a responder la escala de forma individual, a la confidencialidad de la información y a que no había respuestas categorizadas como "buenas" o "malas", sino que se pretendía conocer el funcionamiento psicológico positivo de los jóvenes uruguayos.

\section{Análisis de datos}

El análisis de datos se realizó por medio de los programas SPSS 22.0 y FACTOR 10.8.04 (Lorenzo-Seva \& Ferrando, 2006). El uso del programa FACTOR para el análisis factorial exploratorio (AFE) se debe a la ventaja que ofrece de llevar a cabo el estudio mediante matrices de correlación policóricas, estas se sugieren para los casos en que los ítems presentan un formato de respuesta tipo Likert (Muthen \& Kaplan, 1992). 


\section{Resultados}

En primer lugar, se analizaron los datos descriptivos de los ítems (ver Tabla 1). Se realizaron pruebas de esfericidad de Bartlett y la media de adecuación muestral KMO para conocer si los ítems están lo suficientemente correlacionados. La prueba de Bartlett arroja un índice significativo $(\mathrm{X} 2=3073,7 ; \mathrm{gl}=528 ; \mathrm{p}<.000$ ), lo cual permite rechazar la hipótesis nula; mientras que el índice $\mathrm{KMO}=.898$ indica un nivel de adecuación considerado como muy bueno (Llorente, Ferreres, Hernández, \& Tomás, 2014), lo cual comprueba la pertinencia del uso de la escala para el análisis factorial.

Tabla 1.

Estadísticos descriptivos de los ítems

\begin{tabular}{lcccc}
\hline & $M$ & $D E$ & Asimetría & Curtosis \\
\hline ítem 1 & 3.98 & 1.11 & -1.04 & 0.39 \\
ítem 2 & 3.80 & 1.06 & -0.70 & -0.09 \\
ítem 3 & 3.70 & 1.07 & -0.67 & -0.13 \\
ítem 4 & 3.52 & 1.06 & -0.36 & -0.30 \\
ítem 5 & 3.67 & 1.14 & -0.53 & -0.55 \\
ítem 6 & 3.41 & 1.04 & -0.36 & -0.41 \\
ítem 7 & 3.75 & 1.04 & -0.48 & -0.44 \\
ítem 8 & 3.86 & 1.08 & -0.53 & -0.49 \\
ítem 9 & 3.35 & 0.99 & -0.07 & -0.32 \\
ítem 10 & 3.77 & 1.02 & -0.62 & 0.04 \\
ítem 11 & 4.09 & 1.24 & -1.25 & 0.42 \\
ítem 12 & 3.83 & 1.10 & -0.60 & -0.59 \\
ítem 13 & 4.16 & 1.17 & -1.41 & 1.08 \\
ítem 14 & 3.71 & 1.16 & -0.64 & -0.43 \\
ítem 15 & 3.35 & 1.22 & -0.26 & -0.86 \\
ítem 16 & 3.79 & 1.09 & -0.62 & -0.43 \\
ítem 17 & 3.48 & 1.15 & -0.35 & -0.75 \\
ítem 18 & 3.89 & 1.17 & -0.90 & -0.08 \\
ítem 19 & 3.69 & 1.07 & -0.68 & -0.06 \\
ítem 20 & 3.76 & 1.39 & -0.84 & -0.59 \\
ítem 21 & 4.02 & 1.15 & -1.08 & 0.44 \\
ítem 22 & 3.01 & 1.27 & 0.04 & -1.04 \\
ítem 23 & 4.14 & 1.17 & -1.35 & 0.96 \\
ítem 24 & 4.18 & 1.10 & -1.48 & 1.56 \\
ítem 25 & 3.87 & 1.21 & -0.84 & -0.25 \\
ítem 26 & 3.98 & 1.10 & -0.90 & 0.06 \\
ítem 27 & 1.22 & -1.01 & -0.01 \\
ítem 28 & 3.95 & 1.13 & -0.51 & -0.42 \\
ítem 29 & 1.20 & -0.62 & -0.48 \\
ítem 30 & 1.22 & -0.57 \\
ítem 31 & 1.15 & -0.75 & -0.42 \\
ítem 32 & 1.07 & 0.02 \\
ítem 33 & 1.09 & -0.27 & \\
\hline & 3.77 & & -0.48 & \\
\hline & 3.81 & & \\
\hline
\end{tabular}


Para evaluar la validez de la escala se realizó un análisis factorial exploratorio por medio del método de cuadrados mínimos no ponderados (Pérez, 2004). Este método de estimación es recomendado cuando la muestra es pequeña, en tanto recupera el factor débil en los casos en que el método de máxima verosimilitud falla y el modelo incluye una cantidad mayor de factores (Ximénez \& García, 2005). Los resultados arrojan una solución de siete factores que explican el $72,91 \%$ de la varianza. Cabe destacar que se realizaron los estudios con otros métodos y todos arrojaron una solución factorial de siete dimensiones (ver Tabla 2).

Tabla 2.

Estructura factorial de la escala

\begin{tabular}{|c|c|c|c|c|c|c|c|}
\hline \multirow[t]{2}{*}{ Ítem } & \multicolumn{6}{|c|}{ Factor } & \multirow[b]{2}{*}{7} \\
\hline & 1 & 2 & 3 & 4 & 5 & 6 & \\
\hline 1 & .76 & .20 & .20 & .21 & .05 & .25 & .19 \\
\hline 2 & .25 & .08 & .54 & .18 & .04 & .05 & .08 \\
\hline 3 & .43 & .04 & .09 & .62 & .30 & .15 & -.12 \\
\hline 4 & .23 & .12 & .31 & -.02 & .52 & .15 & .25 \\
\hline 5 & .82 & .15 & .18 & -.01 & .28 & .12 & .29 \\
\hline 6 & .26 & .26 & .11 & .16 & .12 & -.08 & .32 \\
\hline 7 & .30 & .20 & .18 & .74 & .26 & .12 & .27 \\
\hline 8 & .15 & .57 & .19 & .14 & .17 & .15 & .06 \\
\hline 9 & -.09 & .23 & .02 & .05 & .37 & .02 & .08 \\
\hline 10 & .11 & 1.04 & .04 & .01 & .23 & .01 & .12 \\
\hline 11 & .24 & .43 & .82 & .24 & .09 & .27 & .06 \\
\hline 12 & .07 & .53 & .30 & .12 & .28 & .17 & .01 \\
\hline 13 & .28 & .26 & .29 & .29 & .13 & .67 & -.08 \\
\hline 14 & .22 & .19 & .47 & .04 & .25 & .28 & .18 \\
\hline 15 & .23 & .12 & .01 & .47 & .25 & .20 & .06 \\
\hline 16 & .18 & .28 & .51 & .12 & .18 & .14 & .21 \\
\hline 17 & -.01 & .19 & .13 & .12 & .46 & .18 & .23 \\
\hline 18 & .90 & .24 & .22 & .21 & .17 & .22 & .24 \\
\hline 19 & .29 & .04 & .22 & .61 & .17 & .37 & .19 \\
\hline 20 & .77 & .21 & .03 & .24 & .04 & .20 & .27 \\
\hline 21 & .30 & .26 & .35 & .79 & .17 & .25 & .12 \\
\hline 22 & -.16 & .10 & -.01 & .08 & .09 & .10 & -.48 \\
\hline 23 & .31 & .19 & .32 & .22 & -.05 & .71 & .02 \\
\hline 24 & .17 & .36 & .91 & .32 & .08 & .27 & .13 \\
\hline 25 & .14 & .43 & .82 & .25 & .14 & .22 & -.01 \\
\hline 26 & .17 & .33 & .72 & .24 & .22 & .10 & .26 \\
\hline 27 & -.09 & .28 & .17 & .33 & .81 & .35 & .26 \\
\hline 28 & -.07 & .16 & -.01 & .33 & .58 & .33 & .22 \\
\hline 29 & .07 & .02 & .03 & .07 & .31 & .76 & .03 \\
\hline 30 & -.07 & .09 & .33 & .11 & .82 & .32 & .37 \\
\hline 31 & .01 & .06 & -.01 & .12 & .08 & .07 & .66 \\
\hline 32 & .28 & .01 & .10 & .63 & .16 & .28 & .26 \\
\hline 33 & .11 & .12 & .26 & .78 & .21 & .18 & -.02 \\
\hline
\end{tabular}

Método de extracción: cuadrados mínimos no ponderados. Método de rotación: Equamax con normalización Kaiser. 
Se realizó un análisis paralelo basado en el mínimo rango del factor (Timmerman \& Lorenzo Seva, 2011), que informa de una única dimensión de segundo orden, lo cual concuerda con los análisis originales de la escala. El gráfico de sedimentación confirma la estructura de segundo orden (ver Figura 1).

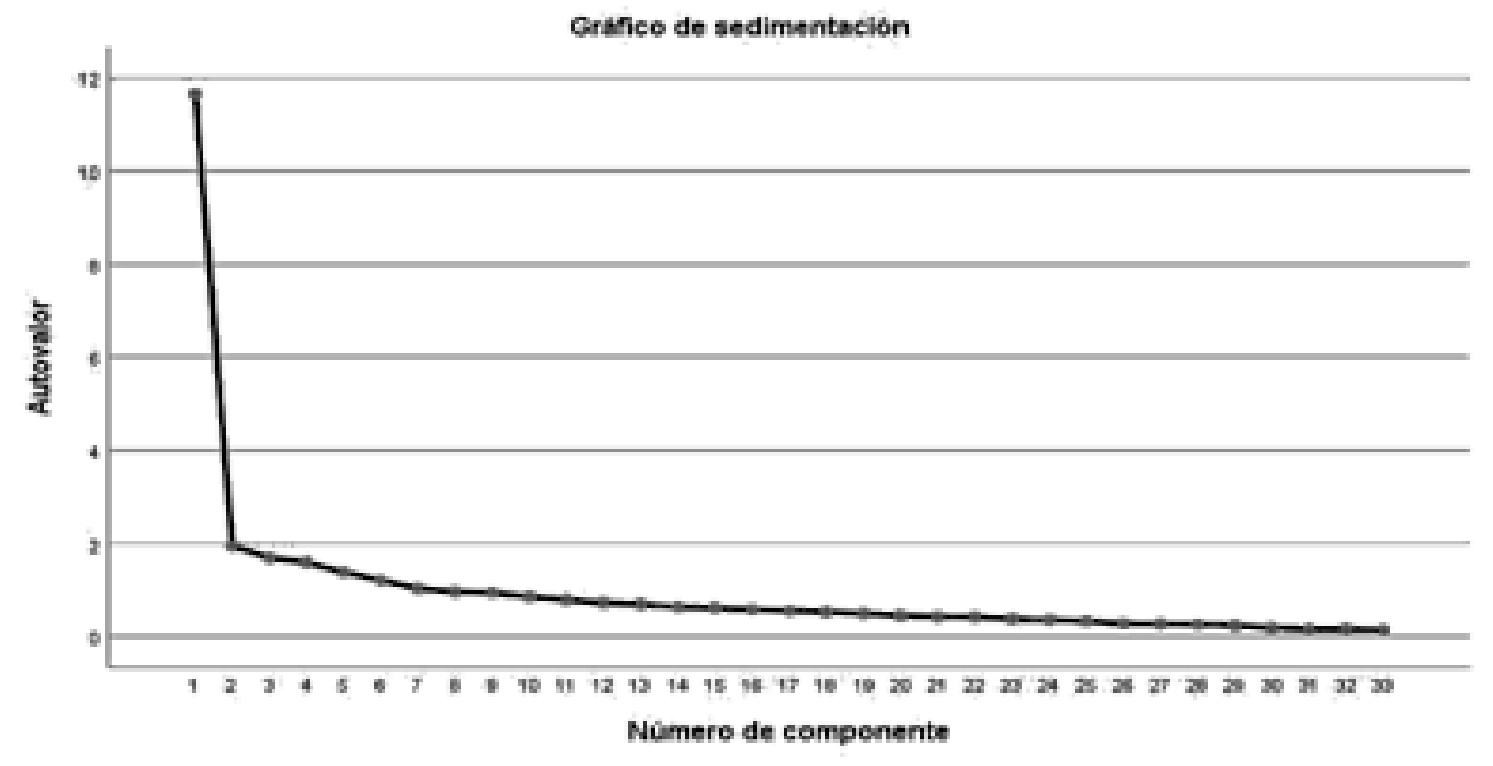

Figura 1. Gráfico de sedimentación de escala FPP.

A continuación, se procede a la descripción e interpretación de los factores emergentes, analizando los resultados obtenidos a la luz de la teoría sobre los constructos implicados (MartínezArias, Hernández-Lloreda, \& Hernández-Lloreda, 2006). Los siete factores emergentes para esta muestra se agrupan de la siguiente manera: F1 (autoestima) ítems 1, 5, 18, 20; F2 (autonomía) ítems 8, 10, 12; F3 (propósito vital) ítems 2, 11, 14, 16, 24, 25, 26; F4 (optimismo/disfrute) ítems 3, 7, 15, 19, 21, 32, 33; F5 (curiosidad) ítems 4, 9, 17, 27, 28, 30; F6 (humor) ítems 13, 23, 29; F7 (dominio del entorno) ítems 6, 22, 31.

Por otra parte, los valores $\alpha$ de Cronbach para el total de participantes en los siete factores emergentes fueron: factor 1 se corresponde con autoestima ( $\alpha .84)$; factor 2 con autonomía $(\alpha .87)$; factor 3, propósito vital ( $\alpha .82)$; factor 4, optimismo/disfrute $(\alpha .82)$; factor 5, curiosidad $(\alpha .83)$; factor 6, humor ( $\alpha .83)$; y factor 7, dominio del entorno $(\alpha .87)$. 
Tabla 3.

Matriz de correlaciones entre los factores

\begin{tabular}{|c|c|c|c|c|c|c|c|}
\hline & Autoestima & Autonomía & Prop. vital & $\begin{array}{l}\text { Optimismo } \\
\text { /disfrute }\end{array}$ & Curiosidad & Humor & $\begin{array}{r}\text { Dom. } \\
\text { entorno }\end{array}$ \\
\hline Autoestima & 1.00 & .40 & .59 & .66 & .46 & .57 & .27 \\
\hline Autonomía & & 1.00 & .54 & .41 & .46 & .34 & .29 \\
\hline Propósito Vital & & & 1.00 & .68 & .71 & .63 & .33 \\
\hline $\begin{array}{l}\text { Optimismo/disf } \\
\text { rute }\end{array}$ & & & & 1.00 & .64 & .71 & .33 \\
\hline Curiosidad & & & & & 1.00 & .58 & .39 \\
\hline Humor & & & & & & 1.00 & .20 \\
\hline Dom. entorno & & & & & & & 1.00 \\
\hline
\end{tabular}

Se analizaron estadísticos descriptivos (media, desviación típica, mínimo, máximo, asimetría y curtosis) de los 7 factores resultantes del instrumento (ver Tabla 4).

Tabla 4.

Estadísticos descriptivos para la muestra de adolescentes

\begin{tabular}{lcccccc}
\hline & $M$ & $D E$ & Asimetría & Curtosis & Mínimo & Máximo \\
\hline Autoestima & 16.21 & 4.144 & -1.218 & 0.945 & 4 & 20 \\
Autonomía & 10.73 & 2.466 & -0.264 & 0.41 & 5 & 15 \\
Propósito Vital & 27.09 & 7.29 & -0.955 & -0.006 & 9 & 35 \\
Optimismo/Disfrute & 26.48 & 7.19 & -0.517 & -1.022 & 12 & 35 \\
Curiosidad & 22.15 & 5.051 & -0.583 & 0.23 & 10 & 30 \\
Humor & 11.61 & 3.445 & -1.108 & 0.304 & 3 & 15 \\
Dominio Entorno & 9.03 & 2.506 & -0.442 & 0.514 & 3 & 15 \\
\hline
\end{tabular}

Como se puede observar, estos resultados sugieren valores adecuados para los factores emergentes. Por último, se estimó la fiabilidad de la escala obteniendo un índice de Alfa de Cronbach de .93, siendo un índice muy aceptable.

Los índices de ajuste de la escala con el presente modelo de agrupamiento de los ítems en siete dimensiones de acuerdo con su carga factorial obtuvieron valores aceptables: $\chi 2=5222.96$, $\mathrm{p}=.00, \mathrm{CFI}=.991, \mathrm{TLI}=.97$, y RMSEA $=.09$, con adecuada carga factorial de los ítems en los factores encontrados. Esta solución factorial explica el $62,15 \%$ de la varianza, lo cual es considerado como satisfactorio, ya que en ciencias sociales se toma como referencia mínima el 60 $\%$ de la varianza total (Hair, Anderson, Tathan, \& Black, 2004). 


\section{Discusión}

Para el presente estudio se planteó el objetivo de analizar las propiedades psicométricas de la escala de Funcionamiento Psicológico Positivo (Merino \& Privado, 2015) en una muestra de adolescentes uruguayos de población general. El análisis psicométrico realizado arroja saturaciones factoriales estadísticamente significativas $(\mathrm{p}=.00)$ y considerablemente elevadas (> .30). La consistencia interna de los factores emergentes es muy buena.

El resultado de este estudio arroja una solución factorial diferente a la versión original en esta muestra de estudio, siendo que de los 11 factores que indican los autores para esta muestra se observa un total de 7 factores con valores considerados como muy satisfactorios; mientas que sí se replica el constructo de segundo orden.

Se realizó el AFE arrojando una estructura factorial de 7 factores que, de acuerdo a la concordancia teórica de la expresión de los ítems, se corresponden con: F1 autoestima; F2 autonomía; F3 propósito vital; F4 optimismo/disfrute; F5 curiosidad; F6 humor; F7 dominio del entorno con valores de $\alpha>.80$ en todas las dimensiones.

La diferencia en la solución factorial se asemeja a los resultados del único estudio publicado en población adolescente, que reporta una distribución de la escala en 4 dimensiones con una varianza explicada del 56,83 \% (González et al., 2018). Este estudio preliminar podría estar colaborando a la conclusión de dicho estudio, en tanto que las diferencias en la estructura factorial de la escala original pueden verse afectadas por la edad.

Los valores de $\alpha(>.80)$ para la muestra fueron muy aceptables. La escala resulta ser un instrumento fiable para la población adolescente de Uruguay.

Entendemos que es importante contar con instrumentos de medición válidos para nuestra población, que evalúen el funcionamiento psicológico positivo y permitan abordar las áreas de recursos personales con los que cuentan las personas, en el entendido de que se trata de capacidades entrenables en los diferentes abordajes terapéuticos.

Por otra parte, cabe destacar que se trata de un área de trabajo novedosa dentro de la disciplina psicológica por lo que se enriquece y beneficia de instrumentos fiables para su medición, tanto en la etapa diagnóstica como de tratamiento.

Los resultados preliminares del presente estudio indican que la escala del FPP es un instrumento válido para evaluar a los adolescentes uruguayos de población general. No obstante, este estudio presentó las siguientes limitaciones: en primer lugar, el tamaño de la muestra, el reclutamiento de los participantes fue por medio del método de bola de nieve, lo cual condiciona la selección e impide el monitoreo de las respuestas, pudiendo estar afectadas tanto por el tiempo de respuesta como por condiciones ambientales.

Considerando las limitaciones expresadas y con el fin de obtener mayor sustento a la fiabilidad del instrumento sería importante continuar estudiando las propiedades psicométricas en población adulta, así como ampliar el número de participantes. Al mismo tiempo, sería deseable la aplicación de la escala a la población de nivel socioeconómico vulnerable para comparar los resultados obtenidos. 


\section{Referencias}

Alvaro, C., Lyons, R., Warner, G., Hobfoll, S., Martens, P., Labonté, R., \& Brown, R. (2010). Conservation of recurces theory and reseach use in health systems implementation. Science, 5(79), 1-20. doi: 10.1186/1748-5908-5-79.

Gaete, V. (2015). Desarrollo psicosocial del adolescente. Revista Chilena de Pediatria, 86(6), 436443. doi: 10.1016/j.rchipe.2015.07.005.

González, C., Andrade, P., \& Jimenez, A. (1997). Recursos psicológicos relacionados con el estres cotidiano en una muestra de adolescentes mexicanos. Salud Mental, 20(1), 27-35.

González, N., Torres, M., González, S., \& Ruiz, A. (2018). Propiedades Psicométricas de la escala de Funcionamiento Psicológico Positivbo en Adolescentes. Revista de Psicología de la Universidad Autónoma del Estado de México, 7(14), 80-97.

Hair, J., Anderson, R., Tathan, R., \& Black, W. (2004). Analisis Multivariante. Madrid: Perarson.

Hobfoll, S. (1989). Conservation of resources.A new attempt at conceptualizing stress. Americna Psychologist, 44(3), 513-524.

Hobfoll, S. (2002). Social and psychological resources and adaptation. Review of General Psychology, 6(4), 307-324.

Llorente, S., Ferreres, A., Hernández, A., \& Tomás, I. (2014). El análisis factorial exploratorio de los items: una guía práctica, revisada y actualizada. Anales de Psicología, 30(3), 1151-1169. doi: 10.6018/analesps.30.3.199361.

Lorenzo-Seva, U., \& Ferrando, P. J. (2006). FACTOR: A computer program to fit the exploratory factor analysis model. Behavior Research Methods, 38(1), 88-91. doi: 10.3758/bf03192753

Muthen, B., \& Kaplan, D. (1992). A comparison of some methodologies for the factor analysis of non-normal Likert variables: A note on the size of the model British Journal of Mathematical and Statistical Psychology, 45(1), 19-30. doi: 10.1111/j.2044-8317.1992.tb00975.x

Martínez-Arias, M., Hernández-Lloreda, M., \& Hernández-Lloreda, M. (2006). Psicometría. Madrid: Alianza.

Merino, D., \& Privado, J. (2015). Positive Psychological Funcioning. Evidence for a new construct and its measurement. Anales de Psicología, 31(1), 45-54.

Merino, D., Privado, J., \& Gracia, Z. (2015). Validacion Mexicana de la escala de Funcionamiento Psicológico Positivo: perspectivas en torno al estudio del bienestar y su medida. Salud Mental, 38(2), 109-115.

Muratori, M., Zubieta, E., Ubillos, S., González, J., \& Bobowik, M. (2015). Felicidad y Bienestar Psicológico:estudio comparativo entre Argentina y España. Psykhe, 1-18 doi: 10.7764/psykhe.24.2.900.

Oliveira, E., Merino, M., Privado, J., \& Almeida, L. (2017). Escala de Funcionamento Psicológico Positivo: Adaptação e Estudos Iniciais de Validação em Universitários Portugueses. Revista Iberoamericana de Diagnóstico y Evaluación e AvaliaçãoPsicológica, 3(48), 151-162. doi:10.21865/RIDEP48.3.13.

Padrós, F., \& Rivera, M. (2014). Bienestar psicológico, recursos psicológicos y calidad de vida ¿conceptos afines y diferentes? Eurythmie, 1(1), 57-63

Pérez, C. (2004). Técnicas de Análisis Multivariantes de Datos. Aplicaciones con SPSS. Madrid: Pearson.

Rueda, B., \& Pérez, A. (2005). Estudio de la competencia percibida procesos de valoración y afrontamiento ante un supuesto problema cardiovascular. Revista de Psicopatologia y Psicologia Clínica, 10(3), 193-204.

Ryff, C. (1989). Beyong ponce de leon and life satisfaction:New directions in quest of successful aging. International Journal of Behavioral Development, 12(1), 35-55. 
Timmerman, M. E., \& Lorenzo-Seva, U. (2011). Dimensionality assessment of ordered polytomous items with parallel analysis. Psychological Methods, 16(2), 209-220. doi:10.1037/a0023353

Tomyn, A., Weinberg, M., \& Cummins, R. (2015). Intervention Efficacy Among "At Risk" Adolescentes: a test of subjetive wellbeing homestasis theory. Social Indicators Research, 120, 883-895.

Vázquez, M., Rivera, M., \& Quintanilla, R. (2001). Recursos psicológicos y resiliencia en niños de 6, 8 y 10 años de edad. Revista de educación y desarrollo, 17, 33-41.

Ximénez, M., \& García, A. (2005). Comparación de los métodos de estimación de máxima verosimilitud minimos cuadrados no ponderados en el análisis factorial mediante simulación Monte Carlo. Psicothema, 17(3), 528-535.

Contribución de los autores: a) Concepción y diseño del trabajo; b) Adquisición de datos; c) Análisis e interpretación de datos; d) Redacción del manuscrito; e) revisión crítica del manuscrito. M. P. ha contribuido en a, b, c, d y e.

Editora científica responsable: Dra. Cecilia Cracco 